\title{
Erratum to: Mycena kentingensis, a new species of luminous mushroom in Taiwan, with reference to its culture method
}

\author{
Yu-Shen Shih • Chi-Yu Chen • Wen-Wen Lin • Hsiao-Wei Kao
}

Published online: 28 January 2014

(C) German Mycological Society and Springer-Verlag Berlin Heidelberg 2014

\section{Erratum to: Mycol Progress}

DOI 10.1007/s11557-013-0939-x

The original version of this article contained two mistakes.

1) Section "Introduction", the first paragraph, should read:

The luminous fungi are capable of emitting light from their fruiting bodies, mycelia, or both (Desjardin et al. 2008; Mori et al. 2011). Until now, 73 species of luminous fungi (Basidiomycota) have been recorded in the world. Among them, 43 species belong to 17 sections of Mycena (Desjardin et al. 2008, 2010; Capelari et al. 2011; Aravindakshan et al. 2012).

2) Section "Taxonomy", the third paragraph, should read:

Holotype examined: Sheding Nature Park of Kenting National Park in Taiwan $\left(21^{\circ} 57^{\prime} 25.81^{\prime \prime} \mathrm{N}, 120^{\circ} 49^{\prime} 14.98^{\prime \prime} \mathrm{E}\right.$, elev. ca. $202 \mathrm{~m}$ ), solitary or scattered on decaying woody shells of unknown dicotyledonous plants. Specimens were collected by Y-S Shih on 11 Nov. 2011 and deposited in the National Museum of Nature Science of Republic of China, Taichung, Taiwan (TNM) under the voucher number of F0026642.

The online version of the original article can be found at http://dx.doi.org/ 10.1007/s11557-013-0939-x.

Y.-S. Shih • H.-W. Kao $(\bowtie)$

Department of Life Sciences, National Chung Hsing University,

Taichung 40227, Taiwan

e-mail: hkao@dragon.nchu.edu.tw

\section{C.-Y. Chen}

Department of Plant Pathology, National Chung Hsing University, Taichung 40227, Taiwan

W.-W. Lin

Department of Marine Biotechnology, National Kaohsiung Marine University, Kaohsiung 81157, Taiwan 